\title{
Unusual pulmonary complication of ulcerative colitis with a rapid response to corticosteroids:
} case report

\author{
G A L Hilling, D A F Robertson, A H Chalmers, H S Rigby
}

\begin{abstract}
Extraintestinal features of ulcerative colitis are well recognised. Pulmonary complications include pulmonary vasculitis, fibrosing alveolitis, asthma, and chronic bronchial suppuration. A case is described of a patient with longstanding quiescent ulcerative colitis who developed a life threatening pulmonary complication, which is extremely sensitive to corticosteroid treatment.

(Gut 1994; 35: 847-848)
\end{abstract}

\section{Case report}

A 32 year old man presented in 1977 with intermittent diarrhoea, abdominal pain, and rectal bleeding. Investigations included barium enema, colonoscopy, and colonic biopsies, which confirmed the clinical impression of mild total ulcerative colitis. His symptoms responded quickly to treatment with sulphasalazine $1 \mathrm{~g}$ three times a day. There have been two subsequent minor exacerbations of his symptoms, which responded quickly to prednisolone enemas. In 1987, his maintenance treatment for ulcerative colitis was changed to mesalazine $400 \mathrm{mg}$ three times a day. In June 1990, he reported increasing breathlessness on exertion, dry cough, and pleuritic chest pain. There was no change in his bowel habit. On examination, he was febrile with tachycardia and bronchial breathing at both apices. Examination showed some weight loss. Haemoglobin $12.5 \mathrm{~g}$, white cell count $19.3 \times 10 / 1$ (neutrophil leucocytosis), platelet count $518 \times 10 / 1$. C reactive protein 0.11 (normal less than 0.01 ) and plasma

Royal United Hospital, Combe Park, Bath G A L Hilling

D A F Robertson

A H Chalmers

H S Rigby

Correspondence to Dr D A F Robertson, Royal United Hospital, Comb Park, Bath BA1 3NG.

Accepted for publication 20 October 1993

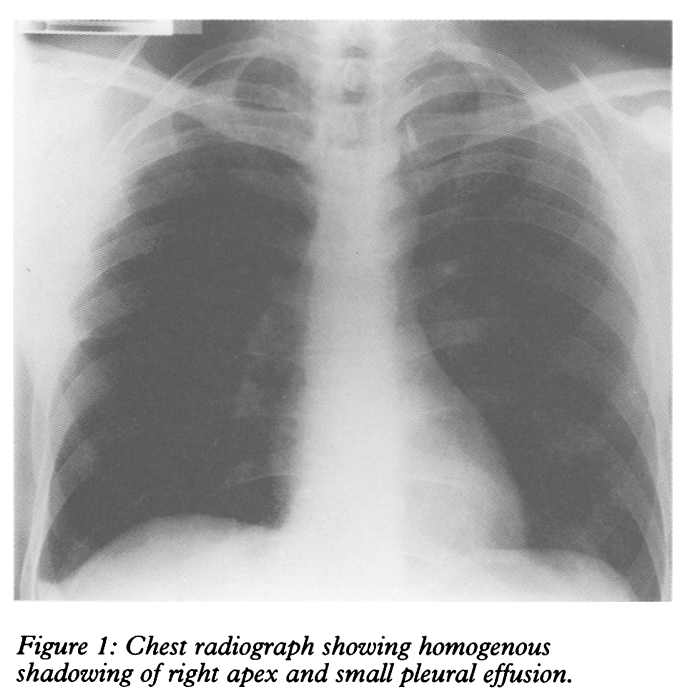

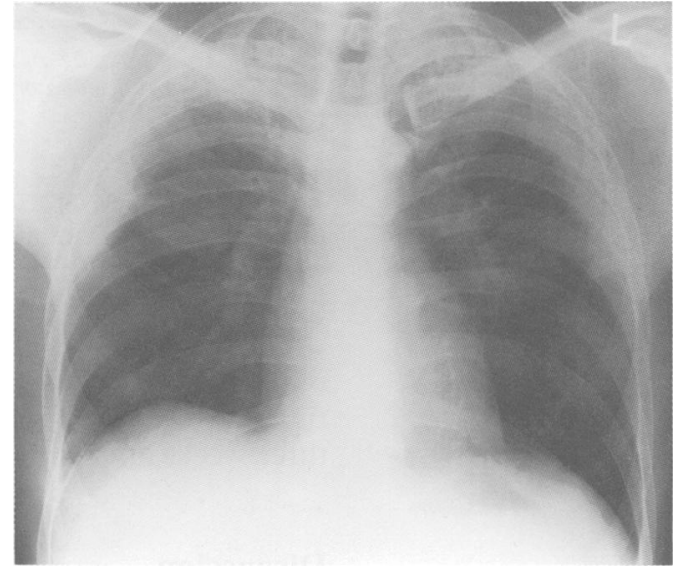

Figure 2: Chest radiograph showing progressive changes.

viscosity raised at $1.93 \mathrm{mp}$. Electrolytes and liver function tests were normal. Autoimmune profile, including antineutrophil cytoplasmic antibodies, and immunoglobulin values were normal. A chest $x$ ray (Fig 1) showed homogenous shadowing at the right apex with a small right pleural effusion. There was no loss of volume of the right lung. Pulmonary function tests showed a restricted pattern (forced vital capacity 3.36 litres, $79 \%$ of predicted, peak expiratory flow $515 \mathrm{l} / \mathrm{min}$ ) and oxygen saturation was reduced to $95 \%$. Carbon monoxide transfer factor was normal $(97 \%$ of expected). A Heaf test and culture of sputum for acid fast bacilli were negative. Bronchoscopy showed no endobronchial lesion, broncho alveolar lavage showed inflammatory changes only, and was negative for tuberculous culture. Transbronchial biopsy was not performed. Over the next four weeks, his chest pain, breathlessness, and weight loss continued with progressive changes on the chest $x$ ray (Fig 2). There was now peripheral consolidation in both upper lobes with air bronchograms, ill defined shadowing in both lower lobes, and considerable loss of volume in both lungs. There were no cavities, septal lines or pleural effusions.

It was then decided to do an open lung biopsy. Histological examination showed an acute and chronic peribronchial and peribronchiolar inflammatory cell infiltrate with associated peribronchiolar fibrosis (Fig 3). Some of the bronchioles showed narrowing of the lumen resulting from bronchiolar inflammation with concentric submucosal fibrous thickening. There was little alveolar cell infiltrate, and no evidence of organising pneumonia. Treatment with prednisolone 


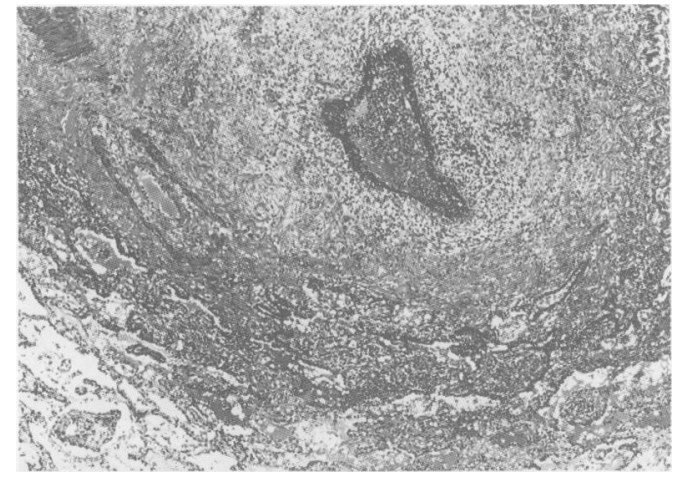

Figure 3: Lung showing an acute and chronic peribronchiolar inflammatory cell infiltrate with peribronchiolar fibrosis.

$40 \mathrm{mg}$ daily was started and he made a complete symptomatic and radiological recovery with return of the chest radiograph and pulmonary function to normal. Oral steroids were withdrawn four weeks later and he remains well while receiving mesalazine three times a day. A steroid inhaler was used for three weeks after withdrawing oral steroids.

\section{Discussion}

Airways involvement in ulcerative colitis, although well reported, is comparatively uncommon. To some extent, this seems to be unrelated to disease activity in the bowel, as pulmonary disease may manifest years after the patient has had colectomy. ${ }^{1}$ The disease process is predominantly obstructive in nature and most patients may present with a large airway chronic suppurative bronchitis and bronchiectasis, fibrotic obliterative bronchitis, concentric bronchiolitis obliterans or small airway changes similar to those seen in smokers. Rare cases of pulmonary vasculitis and apical fibrosis have been described in association with ulcerative colitis. ${ }^{2} 3$

The histological changes seen in our case mainly result from large airway chronic bronchitis with some bronchial fibrosis and obliterative bronchiolitis resulting from concentric submucosal fibrosis. The $x$ ray appearances, pulmonary function tests, and the dramatic response to steroids in our patient is suggestive of bronchiolitis obliterans organising pneumonia ${ }^{4}$ but the lung biopsy showed no evidence of organising pneumonia, in particular no fibrous knots were seen in distal air spaces.

It has been pointed out that the changes in the airways are comparable with those that develop in sclerosing cholangitis in the biliary tree, which is another extraintestinal complication of ulcerative colitis. ${ }^{1}$ In ulcerative colitis, it seems that proctocolectomy has no beneficial effect on the progress of the bronchial disease and, in 15 of 18 previously reported cases, treatment with steroids was beneficial. ${ }^{5}$

There are morphological and developmental similarities between colonic and bronchial epithelium. It has been suggested that there may be a systemic factor responsible for the common response at both epithelial sites in patients with ulcerative colitis or that both the bronchial and colonic epithelium are possibly unduly sensitive to contact with inhaled and ingested irritants. ${ }^{6}$

It is important to recognise the association between steroid responsive lung disease and ulcerative colitis.

1 Corrin B. The Lungs, systemic pathology. 3rd Ed. Vol 5. London: Churchill Livingstone, 1990: 174

2 Isenberg JI, Goldstein H, Korn AR, Ozeran RS, Rosen Y. Pulmonary vasculitis: an uncommon complication of ulcerative colitis. N Engl f Med 1968; 279: 1376-7.

3 Meadway J. Ulcerative colitis, colonic spondylitis and associated apical pulmonary fibrosis. Pro $R$ Soc Med 1974; 67: 16-7.

4 Swinburn CR, Jackson GJ, Cobden I, Ashcroft T, Morritt GM, Corris PA. Bronchiolitis obliterans organising pneumonia in a patient with ulcerative colitis. Thorax 1988; 43: 735-6.

5 Moles KW, Varghese G, Hayes JR. Pulmonary involvement in ulcerative colitis. Br F Dis Chest 1988; 82: 79-83.

6 Higgenbottom T, Cochrane GM, Clark TJH, Turner D, Mittis R, Seymour W. Bronchial disease in ulcerative Mittis R, Seymour W. Bronch
colitis. Thorax 1980; 35: 581-5. 